\title{
The combined effect of soil-applied azadirachtin with entomopathogens for integrated management of western flower thrips Jacinter A. Otieno ${ }^{1}$, Philip Pallmann ${ }^{2}$ and Hans-Michael Poehling ${ }^{3}$
}

\author{
${ }^{1,3}$ Institute of Horticultural Production Systems-Department of Phytomedicine, Leibniz* \\ University Hannover, Herrenhaeuser Strasse 2, 30419 Hannover, Germany \\ ${ }^{2}$ Institute of Biostatistics, Leibniz University Hannover, Herrenhaeuser Strasse 2, 30419 \\ Hannover, Germany
}

\section{*Author for correspondence:}

Hans-Michael Poehling

Tel: +: +49 511762 2641/5343

Fax: +49 5117623015

Email: Poehling@ipp.uni-hannover.de

\begin{abstract}
Performance of soil application of Azadirachtin products with entomopathogens was evaluated as single treatments, in multiple combinations and in different concentrations against the soil stages of Western Flower Thrips, Frankliniella occidentalis (Pergande) in French beans Phaseolus vulgaris L. Treatments consisted of Neem Azal-T solution, Neem pellets, Steinernema carpocapsae (Weiser) Nemastar®, isolates of Metarhizium anisopliae (Metschnikoff) Sorokin (IPP 2539 \& ICIPE-69) and Beauveria bassiana (Balsamo) Naturalis ${ }^{\circledR}$. All treatments were analysed for number of emerging adults, while emerged adults in fungi based treatments were analysed additionally for retarded development of mycosis as a possible cause of secondary mortality. Possible interactive effects in combined treatments were analyzed using a generalised linear model (GLM) approach, and three levels of dose response combinations of the selected treatments were further tested. Bioassay results of the single treatments indicated between $43 \%$ and $60 \%$ reduction in adult emergence with NeemAzal-T solution thus proving to be the most efficient. However, most cadavers with Entomopathogenic Fungi (EPF) treatments showed development of mycosis. Therefore, the reduction in adult emergence attributed to the EPF was altogether $>87 \%$. Combined
\end{abstract}


treatments with Steinernema, Metarhizium (ICIPE), NeemAzal-T and Pellets resulted in total reduction in adult emergence of 95-97\% when late mortality by mycosis was considered. Out of the treatment combinations two showed synergistic, four additive and one an antagonistic response. Combining low concentration of EPN $\left(100 \mathrm{IJ} / \mathrm{cm}^{2}\right)$ with NeemAzal-T resulted in satisfactory control compared to the operational dose of EPN while the highest concentration of $M$. anisopliae ( $10^{8}$ conidia) combined with Steinernema showed the best performance with 74\% reduction in adult emergence. In summary the combined use of Neem Azal-T with the entomopathogens may offer a promising solution by increasing the efficacy and reliability of biocontrol against WFT.

Key words: Frankliniella occidentalis; pupal stage; Neem; Entomopathogenic nematode; Entomopathogenic fungi; Biological control; synergism

Running title: Biocontrol of Soil stages of Western flower thrips

\section{INTRODUCTION}

Western Flower thrips (WFT) is a polyphagous pest, with over 250 different host plants from more than 60 plant families (Morse and Hoddle, 2006). Direct plant damage results in major yield losses (Shipp et al., 2000). WFT is also the key vector of several destructive plant viruses from the genus Tospovirus (Bunyaviridae) (Boonham et al., 2002; Pappu et al., 2009; Webster et al., 2011) such as Tomato Spotted Wilt Virus (TSWV) (Reitz, 2009) and the Impatiens Necrotic Spot Virus (INSV) (Riley and Pappu, 2004).

Because of the severe threat posed by WFT, there has been a heavy reliance on insecticides for its management. This intensive use of insecticides led to development of resistance of WFT against various insecticides (pyrethroids, organophosphates and carbamates) (Gao et al., 2012), WFT have even become resistant to new pesticides such as Spinosad, one of the most effective and Integrated Pest Management (IPM) compatible insecticides (Shan et al., 2012). Resistance in WFT has been reported in Australia (Herron and James, 2005) and southeastern Spain (Bielza et al., 2007). Most troubling to resistance management are recent findings that resistance to insecticides like Acrinathrin and Spinosad does not occur with a fitness cost (Bielza et al., 2008).

Due to increasing concern about the overuse of pesticides, farmers are seeking more environmentally benign methods for controlling arthropod pests and biological control has 
received more attention in the recent past. However, there is no satisfactory and reliable single biological control technique that can effectively control WFT, particularly on high value crops, because of their low damage threshold levels (DeCourcy Williams, 2001; Herrin and Warnock, 2002). Therefore, using a suite of natural enemies or entomopathogens (Arthurs and Heinz, 2006; Brownbridge et al., 2013), which could have additive or synergistic effects, improving the efficacy and reliability of single antagonistic species as well as including lowrisk pesticides with selective application seems to offer a sustainable control strategy.

The WFT life cycle includes the foliar-feeding (adult, first- and second-instars) and soildwelling developmental stages (late second-instars, prepupae, and pupae). Most larvae leave plants as late second instars going to the soil to enter into non-feeding prepupa and pupal stages, with up to $98 \%$ of thrips preferring to pupate in the soil environment (Berndt et al., 2004). An ideal biological control strategy would therefore target both the foliar-feeding and soil-dwelling development stages of the pest (Ansari et al., 2008a). Attention has largely focused on the control of adults and larvae in the crop canopy while few attempts have been made to control soil-dwelling stages of the pest (Berndt et al., 2003; Belay et al., 2005; Ansari et al., 2008a), which constitute significant reservoirs for re-infestation. However given the amount of time spent in the soil, these stages make ideal targets for soil-dwelling antagonists or soil applicable entomopathogens, preferably when they are used as part of an integrated pest-management strategy. The pupal stage in the soil is protected from insecticides sprayed on leaves and stems of the plant, and there are currently no pesticides labelled as drenches to kill pupae in the soil (Ansari et al., 2008a; Cloyd, 2009). Contrastingly, these immobile soil dwelling phases are vulnerable to soil-dwelling predators and pathogens (Ansari et al., 2008a; Steiner et al., 2011; Holmes et al., 2012).

A biorational pesticide potentially suited for an integrated approach to control WFT soil stages is the biopesticide Neem. Soil applied Neem has been shown to be effective against Meloidogyne incognita (Lee et al., 2010), Liriomyza sativae (Hossain et al., 2007), Ceratothripoides claratris (Thoeming and Poehling, 2006) and WFT (Thoeming et al., 2006; Cloyd, 2009). The active ingredient of Neem products, Azadirachtin (AZA), can affect the behavior and physiology of different target insects (Mitchel et al., 2004; Islam et al., 2010). It is registered as an IPM conforming pesticide, with different commercial products available. It has a low risk of pest resistance due to its complex mode of action, and can show low detrimental side effects to a broad range of non-target organisms if properly applied. It is biodegradable in nature (Schmutterer, 1997). However pests with cryptic feeding behavior 
such as thrips are often poorly controlled by foliar application of Neem extracts, because of incomplete contamination. At the same time intensive foliar spraying can increase the risk of toxicity for natural enemies foraging in the crop canopy thus limiting the potential combination of Neem extracts and beneficials in IPM. Soil application can avoid this risk because the systemic properties still affect plant sucking life stages of WFT as well as soil dwelling pupal stages directly (Thoeming et al., 2003).

Over the last two to three decades, EPNs have become increasingly popular as biocontrol agents, especially against soil inhabiting pests (Berndt, 2003). They have a wide host range, and are able to efficiently locate their victims either actively seeking them out or by ambushing and rapidly killing the host. They are safe to vertebrates and bear a low risk of affecting non- target organisms (Shapiro-Ilan et al., 2012). In particular, many products containing S. carpocapsae, are available in the market and their basic potential to control WFT soil stages has already been shown (Premachandra et al., 2003b; Berndt, 2003).

EPFs are most often studied and used to control foliar pests by applying spore solutions to the crop canopy. However, they offer an interesting additional option for controlling soil dwelling pest stages, since the soil is a convenient microclimatic environment for fungal infection. Studies by Ansari et al., (2007; 2008a) have already shown the high susceptibility of thrips pupae to infection by M. anisopliae in various media. Therefore, advances have been made to improve their quality, performance and make them more cost-competitive with chemical pesticides (Lacey et al., 2001).

An option for improving WFT control efficacy on a biorational basis is the combined application of biological agents. Several studies have demonstrated the high efficacy of a combined use of various entomopathogens with Neem products. For example, NeemAzalformulations have been combined with Amblyseius cucumeris and Hypoaspis aculeifer (Thoeming, 2005; Thoeming and Poehling, 2006), Eretmocerus warrae (Kumar et al., 2010), and EPNs (Krishnayya and Grewal, 2002; Meyer et al., 2012). However, the use of different predators and pathogens in combinations requires detailed knowledge about possible interactions to avoid inter- and intra-guild effects while achieving synergism. Different authors have reported various interactive effects. For example, synergistic/additive effects were obtained with combined use of $S$. feltiae and a Neem seed kernel extract, NeemAzal-T (Neemix) (MahMoud, 2007); M. anisopliae and S. kraussei (Georgis, 1997); B. bassiana and S. carpocarpsae (Williams et al., 2013) and M. anisopliae with four EPN species (Ansari et al. 2008b). No study has so far assessed the efficacy of combining EPFs and EPN by soil 
application with specifically designed Neem formulations in controlling the soil stages of WFT.

\section{MATERIALS AND METHODS}

Bean plants and Western Flower Thrips: Dwarf French Beans (Phaseolus vulgaris (L.) var. 'Speedy' (Fabaceae) were pre-germinated for three days before transplanting into plastic seedling trays $(50 \times 30 \times 6.5 \mathrm{~cm})$ at a seeding rate of 50 seeds per tray. A commercial substrate (CS) Frühstorfer Erde, type P (Archut GmbH, Lauterbach-Wallenrod, Germany) was used. The seedlings were left to grow for 6 days until the primary leaf stage under greenhouse conditions of $22 \pm 2{ }^{\circ} \mathrm{C}$ temperature, and $65-75 \%$ relative humidity with a $16: 8 \mathrm{~h}$ light: dark photoperiod. The seedlings were then individually transplanted into $16 \mathrm{~cm}$ diameter plastic pots filled with $600 \mathrm{~g}$ of CS and left to grow for another 6 days in the same greenhouse conditions.

To obtain uniformly aged thrips for the experiments, synchronized rearing of WFT was done on pods of organically grown French Beans in 0.75 L glass jars (Leifheit, Nassau, Germany) in a climate chamber $23 \pm 2^{\circ} \mathrm{C}, 50-60 \%(\mathrm{RH})$ and 16:8 $\mathrm{h}(\mathrm{L}: \mathrm{D})$ photoperiod. The rearing procedure was based on the original protocol by Berndt et al. (2004).

Biopesticide Neem: Two formulations were used. Neem Azal-T solution (1\% Azadirachtin A), mostly suited for hydroponics and soil applications and Neem Azal granules (7 \% Azadirachtin) containing the active ingredient in an inert carrier material (slow release formulation). Both products were obtained from Trifolio-M GmbH and dosed to achieve a basic concentration of $10 \mathrm{mg}$ Azadirachtin/kg soil.

Entomopathogenic Fungi: We tested three isolates of EPFs. M. anisopliae 2539 IPP (access no DSM_Nr 100117), ICIPE-69 (commercialized strain by International Centre of Insect Physiology and Ecology (icipe), for identification characteristics see Niassy et al., 2012b) and B. bassiana (Naturalis ${ }^{\circledR}$-commercial product, BioGard, Grassobbio, Italy). The latter is an emulsifiable suspension (ES) containing $2.3 \times 10^{7}$ conidia/ml. The liquid culture of $M$. anisopliae 2539 IPP was stored in sealed vials at $-20^{\circ} \mathrm{C}$, while the ICIPE 69 culture obtained as dry conidia from ICIPE, was stored at $4^{\circ} \mathrm{C}$.

For the experiments, both $M$. anisopliae strains were reactivated on adult WFT to recover the pathogenic characteristics for WFT (Goettel and Inglis, 1997). Conidia from infected cadavers were isolated onto potato dextrose agar media (PDA), supplemented with peptone 
and yeast extract. This media ensured fast growth and sporulation of the fungi. The petri dishes were then stored in an incubator at $25^{\circ} \mathrm{C}$. Fungal cultures not older than two weeks and grown on artificial media less than three times after isolation from WFT were used for the experiments.

The aqueous suspension of $M$. anisopliae conidia was prepared by scraping off dried conidia from the PDA plates into $15 \mathrm{ml}$ test tubes using a sterile spatula. To determine the number of conidia/g of dry powder, a $0.1 \mathrm{~g}$ sample was aseptically mixed with $100 \mathrm{ml}$ sterile $0.05 \%$ Tween 80 (AppliChem GmbH, Darmstadt, Germany). The solution was vortexed for 5 minutes to break chains or aggregates of conidia to achieve a homogenous suspension. The conidia suspension was adjusted to a final concentration of $10^{7}$ conidia using a Haemocytometer (Fuchs Rosenthal Counting Chamber by Marienfeld, Lauda_Königshofen, Germany) (Goettel and Inglis, 1997). To determine conidia germination five aliquots of a conidia solution diluted to $10^{6}$ conidia/ $\mathrm{ml}$ were added to a Petri dish filled with agar, which was incubated at $25^{\circ} \mathrm{C}$ for 24 hours in darkness. Lacto-phenol cotton blue was used to stop conidial growth and to stain the fungal conidia. Germinated and non-germinated conidia were counted by placing agar cubes under a compound microscope (x400 magnification) and evaluating 100 conidia. Conidium was considered germinated if the germ tube was twice the length of the conidia. Assessing conidia viability before the experiments was useful for adjusting the desired concentration of viable conidia /ml for every bioassay.

Entomopathogenic Nematodes: The S. carpocapsae Nemastar ${ }^{\circledR}$ was obtained as a clay formulation from E-nema GmbH (Raisdorf, Germany) and stored for less than two weeks at $4^{\circ} \mathrm{C}$ until used. Before the experiments, the cold stored nematodes were allowed to acclimatize at ambient room temperature for at least four hours before exposure to WFT. A gram of the product was dissolved in $50 \mathrm{ml}$ de-ionized water and $1 \mathrm{ml}$ of the suspension was mounted on a microscope slide for counting the nematodes. A final concentration of 80,435 infective juveniles in $25 \mathrm{ml}$ of distilled water was prepared by quantification and dilution (Kaya and Stock, 1997; Premachandra et al., 2003b).

Experimental setup (Microcosm): The potted bean plants were separately enclosed in acrylic glass tubes (diameter $10 \mathrm{~cm}$, length $30 \mathrm{~cm}$ ) (AK Kunststoff Technik GmbH, Isernhagen, Germany) serving as microcosm. For ventilation, the open end at the top and four additional holes (diameter $3 \mathrm{~cm}$ ) at the side of the cylinder were closed with thrips proof 
nylon gauze (pore size $64 \mu \mathrm{m}$; Heidland, Gütersloh, Germany). The microcosms were kept in a climate chamber at $23 \pm 2 \mathrm{C}$, RH 50-60 \% and 16:8 - L: D photoperiod.

A cohort of one week old adult WFT from synchronised rearing was anesthetized with a low dosage of carbon dioxide for easy handling. 10 females and 2 males were transferred into a clean Eppendorf tube $(2 \mathrm{ml})$ using a fine hairbrush and sealed. The Eppendorf tube was then hung on the petiole of the bean plant and opened to allow the adults to crawl/fly out of the tube and infest the plant. The edge between cylinder and pot was sealed with parafilm to prevent thrips from escaping. Thrips were allowed to feed and to lay eggs on the plants for three days. Thereafter, the adults were removed from the cylinders by shaking the plant on a white sheet of paper and by using an aspirator. Eight days after introduction of the adults when the larval stage (L2) should have been descending to the soil for pupation (Berndt et al., 2004), the substrate was treated.

\section{Treatments:}

Single compound treatments: All dosages used in the treatments are listed in a supplemental table (S1). For application of NeemAzal-T $0.6 \mathrm{ml}$ of the basic solution containing 1\% AZA was diluted in $100 \mathrm{ml}$ distilled water and drenched while Neem pellets (90 mg) were mixed with the soil then gently watered. For the EPN, 80,435 IJs suspended in $25 \mathrm{ml}$ of distilled water were drenched evenly on top of the soil by using a Pasteur pipette (Premachandra et al., 2003b) giving an amount of $400 \mathrm{IJ} / \mathrm{cm}^{2}$. Afterwards the soil surface was further irrigated with $25 \mathrm{ml}$ of distilled water five minutes after the EPN application to percolate the EPN into the soil layers where the WFT prefer to pupate. To avoid water logging and enhance percolation, plants were not irrigated two days before the EPN application.

For both Metarhizium isolates and for B. bassiana conidia solutions containing $10^{7}$ conidia suspended in $100 \mathrm{ml}$ of Tween $0.05 \%$ were applied to each pot to ensure a total wetting to field capacity of the upper soil layer which should be passed by the descending larvae. During application to the soil, the plant was protected by tissue paper to avoid any contamination. A blank treatment with sterile distilled water only was set up as a control since pre-tests with a set of blanks (0.05\% Tween - EPFs; Blank Neem Azal T; sterile distilled water - EPN) showed no significant differences between the blanks of the compounds used and the water control.

Twelve days after the introduction of adults, the cylinders were removed and the bean plants cut off. Adult emergence rate from the soil was measured as the main parameter for efficacy of the treatments. Based on the photosensitivity of the emerging adults, an emergence 
apparatus called Photoeclector was used. This consisted of an inverted pot of the same dimensions as those used in the experiment. A hole was drilled in its base and a 10ml Pipette tip inserted tightly fitting. A $2 \mathrm{ml}$ Eppendorf tube was placed on top of the pipette tip to capture the emerging adults. For ventilation, four holes, each $2 \mathrm{~cm}$ in diameter were drilled on the sides of the pot and covered with thrips proof nylon screen (64 $\mu \mathrm{m}$ pore size). The two pots were sealed with parafilm to prevent the emerging thrips from escaping.

Daily counts of adults emerging from each pot were recorded for 7 days. Adults from treatments with EPFs (most of which died 2 days later) were incubated at $25^{\circ} \mathrm{C}$ in $55 \mathrm{~mm}$ diameter Petri dishes filled with 5mm plaster of Paris-charcoal (9:1) layer and a Whatman filter paper of the same diameter; $1.5 \mathrm{ml}$ of sterile water was added to maintain humidity. The cadavers of these specimens were later examined under a binocular microscope for spores and hyphae on the surface of the insects to confirm mycosis as the cause of death. A completely randomized design with fifteen replicates per treatment was used.

Combined treatments: For combined treatments we selected the EPF with highest performance M. anisopliae-ICIPE 69, S. carpocapsae (EPN) and both Neem formulations, Neem Azal-T solution and Neem Pellets. Double combinations consisted of treatments with EPF/Neem pellets, EPF/NeemAzal-T, EPN/EPF, EPN/Neem pellets and EPN/NeemAzal-T while triple combinations were composed of EPN/Neem pellets/EPF and EPN/NeemAzalT/EPF. The experimental design and procedure was the same as for the single treatments. The mixing ratios of the combinations are described in Table S1.

Moreover, we tested dose response reactions with three concentration levels for $M$. anisopliae $\left(10^{6}, 10^{7}\right.$ and $10^{8}$ conidia/100ml), S. carpocapsae (100, 400 and $\left.800 \mathrm{IJ} / \mathrm{cm}^{2}\right)$ and NeemAzal-T (basic solution $0.25 \%, 0.5 \%$ and 1\% AZA) applied with the same amounts of drenching water as described with the single treatments above.

Statistical analyses: The total numbers of emerging thrips per pot were analysed using Poisson generalised linear models (GLMs) with logarithmic link function and overdispersion (“quasi-Poisson”) and as explanatory variable the treatment (McCullagh and Nelder, 1989). Means of emergences for single and combined treatments were separated by pairwise comparisons of Poisson means (Tukey test) at a multiple type I error level of 5\%. This test was also used to separate means in the dose response experiment.

As a consequence of the experimental setup, it was not possible to determine the numbers of thrips that were actually exposed to the treatments in the soil. Hence to assess interactions of 
treatments when applied in combinations, again a quasi-Poisson GLM was fit to the counts of emerging adults. The GLM included as explanatory variables the treatment and the date (to acknowledge that the single and combination experiments were run consecutively).

Formal inference about treatment interactions was achieved by testing linear combinations of GLM parameter estimates, using their associated standard errors from the model. The linear combinations were chosen such that they reflected the mixing ratios (Table S1), thereby adopting a straightforward notion of additivity: the expected number of emergences (on the log scale) under additivity was calculated as the sum of fractions of log-emergences in the involved single-treatment groups, where the fractions corresponded to the mixing ratios. Subtracting the observed from the expected log-emergences and then exponentiation of the difference yielded the combination's estimated percentage deviation from additivity.

Whenever a test for a combination produced a p-value below 0.05 , the null hypothesis of additivity was rejected and the interaction was deemed synergistic or antagonistic, depending on the direction of deviation from additivity. For p-values greater than 0.05 the null hypothesis of additivity was retained. All computations were performed in R version 3.1.1 (R Core Team, 2014).

\section{RESULTS}

Single Treatment: All treatments resulted in considerably fewer thrips emergences compared to the control. However, no significant difference in \% reduction in adult emergence among the single treatments was recorded ( $p \geq 0.084$,). The bioassay results indicated between $43 \%$ and $60 \%$ reduction in adult emergence, with NeemAzal-T solution proving to be the most efficient (Fig. 1, left). The daily trend indicated that the treatments particularly reduced the peak of the emergence course, between days 3 and 4, afterwards the numbers of emerged adults were very similar in the control and treatments (Fig.1, right). The daily trend also showed that NeemAzal-T outperformed other treatments having the least number of daily survivals of WFT adults. However, when the mean percentages of individuals being killed after emergence by retarded mycosis were considered, the total survivals from the EPF treatments reduced considerably to 12\% (M. anisopliae-IPP), $11 \%$ (B. bassiana) and 6\% (M. anisopliae-ICIPE) (Fig. 3, left).

Treatment combinations: Based on the results above, S. carpocapsae, $M$. anisopliae ICIPE 69, NeemAzal-T and Neem pellets were further used in various combinations (Table S1). In most cases the combinations of NeemAzal-T and Entomopathogens, or both 
Entomopathogens, resulted in few emergences compared to single treatments, except in combinations with Neem pellets (Fig. 2, left). The course of emergence also showed that combinations with NeemAzal-T solution steadily reduced the WFT population from the second day onwards in contrast to combinations with Neem pellets that took longer to achieve similar reductions in adult emergence (Fig. 2, right).

The combined application of EPN + EPF + NeemAzal-T (65\% mean emergence) (Fig. 2, left) was more effective as compared to treatment with EPN + EPF + Neem Pellet $(p=0.007$ from Tukey's test) but not significantly different from combined application of EPN + NeemAzal$\mathrm{T}(p=0.998), \mathrm{EPN}+\operatorname{EPF}(p=0.996)$ and Neem Azal-T $+\operatorname{EPF}(p=0.463)$. The combined application of Neem Pellets + EPF or EPN were significantly different from combined application of EPN + EPF, NeemAzal-T + EPN and EPN + EPF + NeemAzal-T $(p<0.001)$. In EPF combinations, total survivals were again considerably reduced by the late mycosis effect in all the relevant treatments to between $10 \%$ and $14 \%$ in combinations with Neem pellets while NeemAzal-T resulted in 3- 5\% adult survivals (Fig. 3, right).

Interaction Effects: When the interaction effects were determined, most combined treatments resulted in additive effects (Table 1). However, the double (EPN + EPF) and the triple combinations (EPN + NeemAzal-T +EPF) resulted in synergistic responses. On the other hand, combined application of EPN + Neem pellets showed additivity towards antagonism and EPF + Neem pellets gave a less than additive or an antagonistic response (Table 1).

Dose response: For EPF, a clear dose response relation could be observed for all combinations with Neem Azal-T and the EPN (Fig. 4 - left). The highest concentration of $10^{8}$ conidia/100ml combined with Steinernema showed the best performance with $74 \%$ reduction in adult emergence. Significant differences were recorded between EPF $10^{8}$ conidia/100ml + EPN and combinations with the lowest concentration $(p<0.001)$.

All treatments with NeemAzal-T applied with 0.25 and $0.5 \mathrm{mg} / \mathrm{kg}$ and combined either with EPN or EPF had no significant differences in thrips emergence with a mean reduction of approximately 65\%. However, when the dosage was increased to $1 \mathrm{mg} / \mathrm{kg}$ with both Entomopathogens, there were significant differences between NeemAzal-T 1mg/kg + EPN and NeemAzal-T $0.5 \mathrm{mg} / \mathrm{kg}+\mathrm{EPF}(p=0.0111)$ and NeemAzal-T $0.25 \mathrm{mg} / \mathrm{kg}+\mathrm{EPF}$ $(p=0.0043)$. Meanwhile NeemAzal-T 1mg/kg + EPF differed significantly from NeemAzal-T 
$0.5 \mathrm{mg} / \mathrm{kg}+\mathrm{EPF}(p=0.0181)$ and NeemAzal-T $0.25 \mathrm{mg} / \mathrm{kg}+\mathrm{EPF}(p=0.0073) . \quad$ (Fig. 4 middle).

Dose - response evaluation of EPN with 100, 400 and $800 \mathrm{IJ} / \mathrm{cm}^{2}$ and combinations with EPF and Neem Azal-T did not show a consistent and significant tendency. Except between 100 $\mathrm{IJ} / \mathrm{cm}^{2}+\mathrm{EPF}$ and $800 \mathrm{IJ} / \mathrm{cm}^{2}+$ NeemAzal-T ( $\left.\mathrm{p}=0.0219\right)$. (Fig. 4 - right).

\section{DISCUSSION}

This is the first study combining entomopathogenic nematodes and fungi by soil application with specific Neem formulations for controlling the soil stages of WFT in greenhouse conditions. Neem-Azal formulations and the other antagonists were applied when late L2 were descending to the soil to pupate, mimicking the natural conditions where thrips larvae leave the plants to pupate in the soil (Premachandra et al., 2003b).

Regarding the effect of single compound treatments on F. occidentalis, NeemAzal-T was most efficient. The high efficacy of Neem solutions was also shown by the triple treatment combination; it caused the highest \% reduction in adult emergence. This effect was only exceeded when secondary mortality effects of the EPF based treatments were considered. Contrastingly, the nematodes gave low efficacy despite their active host-finding of infective juveniles being presumed better suited for infecting the cryptic pupal stage of WFT in the soil as opposed to the conidia of EPF which depend on contact by chance either from thrips movement or passive distribution with water in the soil (Williams et al., 2013).

Despite having the same active ingredients and concentrations, NeemAzal-T outperformed Neem pellets. The better performance of AZA applied as solutions in contrast to the formulation in the pellet carrier material could be explained by the specific release characteristics of the Neem pellets, which are formulated for a slow and continuous release of AZA in the rhizosphere for systemic translocation in the plant but yielding too low concentrations in soil for effective contamination of thrips stages. A short-term influence but with high concentration, as achieved by the NeemAzal-T solution, should be more effective. It is possible that the efficacy of the pellets could be enhanced by strongly increasing the pellet concentration in the soil.

Among the three EPFs, B. bassiana was out-performed by the two M. anisopliae strains both in reducing adult emergence and in the late mycosis effect. A poor performance by $B$. bassiana was reported by Skinner et al. (2012) where BotaniGard (a commercial product of B. bassiana) resulted in only $15 \%$ thrips control. In addition, drenching $M$. anisopliae strain V275 yielded higher mortality (84-93\%) than B. bassiana (54-84\%) (Ansari et al., 2008a). 
Jacobson et al. (2001) used B. bassiana as a foliar spray and could reduce thrips population by $65 \%$ to $87 \%$. The commercial product, Naturalis, we used is designated for foliar spray applications and not for soil treatments. Therefore it could be that an insufficient number of conidia reached and adhered to the soil stages of WFT.

Between the two strains of $M$. anisopliae, the ICIPE-69 strain was more efficacious. This could be related to the higher viability of conidia the ICIPE strain consistently showing a viability of $96 \%$ of the conidia compared to only $88 \%$ of the IPP strain. The high efficacy of ICIPE-69 strain to $2^{\text {nd }}$ larval instars of WFT was previously reported by Niassy et al. (2012b), who compared 10 isolates of M. anisopliae, 8 isolates of B. bassiana, and the ICIPE-69 strain which produced the most conidia and therefore proved to be especially virulent.

The soil stages of WFT are most vulnerable for the soil dwelling nematodes (Buitenhuis and Shipp, 2005). We selected $S$. carpocapsae for the studies even though according to Premachandra et al., (2003b), S. feltiae proved more virulent than S. carpocapsae. The latter is an ambush forager which remains near the point of application and searches for its host mostly on the surface while the former combines both ambush and cruiser (actively searching for the hosts in the soil) modes of hunting.

The highest efficacy with EPN could be obtained, if the nematodes are present in the soil when the L2 is moving into the soil to pupate (Premachandra et al. 2003b). This increases the chances of the infective juveniles coming into contact with the thrips, as the nematodes need some time to locate the thrips and the encounter rates are higher when the thrips are still moving in the soil. The commercial strain of S. carpocapsae (Nemastar) caused only $43 \%$ reduction in thrips emergence with the applied dose rate of $400 \mathrm{IJ} / \mathrm{cm}^{2}$. This result is comparable to findings by Premachandra et al. (2003b) who achieved 40-45\% using the same strain at a dose rate of $300 \mathrm{IJ} / \mathrm{cm}^{2}$ of soil. Further studies (see for instance Premachandra et al., 2003b; Ebssa et al., 2004) clearly showed that the strain, times of application as well as the concentration of the nematodes are key determinants of efficacy of the EPNs. The 400 $\mathrm{IJ} / \mathrm{cm}^{2}$ application rate used here, has been shown to be the most effective dose rate (Ebssa $e t$ al., 2001).

The aim of combining different control agents was either to achieve higher efficacies or an increase in reliability. Two control agents applied together may act independently and be directed at different targets in a given host; hence, their effect would be simply additive. However, they may also complementarily improve sensitivity of the target organism and ideally interact synergistically. On the other hand, other competitive interactions are possible leading to antagonistic effects. Our findings showed that combined application of NeemAzal- 
T enhances the efficacy of Entomopathogens. The combined treatments revealed that the double combinations of the two Entomopathogens (EPF + EPN) and the triple combinations of the two Entomopathogens with NeemAzal-T (EPF +EPN +Neem Azal T) were the most efficient with synergistic effects. We hypothesize that Neem weakened the WFT by being a physiological stressor or behavioural modifier, thereby predisposing them to the microbes or reducing the defense response of the thrips making them an easier target for nematode penetration and more susceptible to the fungi. Akbar et al. (2005) also hypothesised that the prolonged intermoult period of insect larvae by the growth regulating action of azadirachtin may give time for the establishment and penetration of fungal conidia through the insect's cuticle.

Evidence for the synergistic interaction of Neem with B. bassiana against Army worms, Spodoptera litura (Fabricius) has been shown by Mohan et al. (2007). Islam et al. (2010) observed that combined use of $0.05 \%$ Neem and $10^{7}$ conidia $/ \mathrm{ml} \mathrm{B}$. bassiana resulted in $97.2 \%$ nymphal mortality of B. tabaci (Gennadius). This caused $20.5 \%$ more nymphal mortality than individual treatments. Nymphal mortality of $90 \%$ of whitefly Bemisia argentifolii (Bellows \& Perring) was obtained when Paecilomyces fumosoroseus (Wize) and azadirachtin were combined (James, 2003). Also, out of 25 treatment combination between NeemAzal-T 5\% and S. carpocapsae by MahMoud, (2007) the responses were 19 synergistic, 1 additive, none antagonistic and 5 without response. In this study, combined treatments resulted not only in increased mortality, but also a faster onset of thrips mortality. The number of cadavers that showed mycosis also increased as compared to treatments with single EPF isolates. The larvae migrating in to the soil might have acquired the inoculum but were more vulnerable to the fungi by the additional stress factor. Alternatively, immature stages might have acquired in the combined treatments relatively few conidia but since mortality is dose related, infection took longer to develop, therefore more individuals survived through to adult stages but succumbed thereafter (Ansari et al., 2008a). Speculating about long-term effects in natural populations conidia from infected thrips could serve as source of secondary inoculum for the spread of fungal infection in the insect population (Mohan et al., 2007). Adding the late effect of the fungi, the double (EPF and EPN) and the triple (EPF, NeemAzal-T and EPF) combinations reduced survivals to $3 \%$. Since the triple combination did not give an extraordinary performance, taking costs into account, the use of EPN and EPF would be preferred.

With respect to nematode-fungi interaction the special mutualistic association of the EPN with bacteria of the genus Xenorhabdus for Steinernematidae, should be considered. The 
symbionts inhibit host immune reactions and kill the host rapidly (Lacey et al. 2001). Successful EPF infection needs a sequence of steps: attachment to the host cuticle, germination, penetration, growth and reproduction of the fungus. It kills the host by depletion of nutrients, digestion of tissue or release of toxins. Thrips already infested by the quick acting nematodes is less mobile and because of the mentioned symbiotic activity more susceptible to the fungi and likewise thrips infected by the fungi should be a weak and easy target for the nematodes thus explaining synergism.

Regarding the dose-response experiments first, a clear relation between dose and efficacy was seen for the EPF, hence increasing the conidia concentration of EPF in the soil would be one option for optimization of efficacy. Moreover, it was obvious that at every dose the combination with EPN consistently performed better than its counterpart with Neem. For instance, a more noticeable significant difference was recorded between the highest EPF concentration $\left(10^{8}\right.$ conidia $)+$ EPN and the lowest $\left(10^{6}\right.$ conidia $)+$ EPN $(p<0.001)$ than its counterpart $10^{8}$ conidia + NeemAzal-T and $10^{6}$ conidia + EPN $(p<0.025)$ and $10^{6}$ conidia + NeemAzal-T $(p<0.005)$. While many authors have reported positive interaction between the two entomopathogens (Georgis, 1997; Ansari et al. 2008b; Williams et al., 2013). Mohan et al. (2007) reported that Neem oil caused general delay in conidial growth of B. bassiana. In addition, in neem sensitive isolates, the growth of $B$. bassiana was decreased but not totaly inhibited resulting in an antagonistic effect. The highest nymph mortality of B. tabaci was recorded when $B$. bassiana was topically applied with drenching application of neem (Islam et al. 2011). Also Niassey et al. (2012a) reported that Azadirachtin in high dosage could have negative effect on sporulation and vegetative growth of $M$. anisopliae-ICIPE 69. However this could also be due to overestimation of toxicity of pesticides usually a phenomenone observed when pesticides are sprayed on rather inert material than on plant substrate. Plants enzymes can detoxify the pesticide or sequestor it in its waxy leaf cuticle making it less available to natural enemies (Desneux et al. 2005; 2006a).

Our findings provide evidence of interaction between Azadirachtin based insecticides and the Entomopathogens, which allows use of NeemAzal-T with reduced rates, hence also improving the economic trade-off. However we must admit that a detailed economic valuation was not the aim of this study. Reduction of the operational dose of NeemAzal-T to $0.25 \%$ or $0.5 \%$ with EPN may be equally effective or even better than a full recommended dose. Surprisingly the dose response for EPN unlike in NeemAzal-T and EPF was not clear. The simultaneous application of the EPF and NeemAzal-T might have had an effect on the nematodes such that an increase in concentration didn't cause a corresponding decrease in 
adult emergence. Although reports about compatibility between Neem formulations and EPNs have been recorded, Meyer et al., (2012) reported subtle effects of NeemAzal-U on the plant parasitic nematode $M$. incognita. Also a soap surfactant used for application of neem oil caused 23-25\% mortality of S. feltiae (Krishnayya and Grewal, 2002).

Conversely, combining very low concentrations of EPN $\left(100 \mathrm{IJ} / \mathrm{cm}^{2}\right)$ with NeemAzal-T resulted in satisfactory control compared to the operational dose of EPN. Practically, EPNs are used against soil-dwelling stages of WFT, and the dose rates presently needed for sufficient control are not economical (Premachandra et al., 2003a). Therefore, this study provides further validation that low doses of EPN can be used in combination with other Biocontrols to offer more satisfactory results..

\section{CONCLUSIONS}

The combined use of the soil applied azadirachtin formulations NeemAzal-T and Neem pellets with the EPN S. carpocapsae (commercial product Nemastar ${ }^{\circledR}$ ), and EPF (non commercial isolate of $M$. anisopliae ICIPE-69) substantially improved efficacy of controlling the soil dwelling stages of WFT in comparison to the use of the individual components. In all the EPF based combinations survivals ranged between $14 \%$ to $3 \%$ when considering secondary mortality due to mycosis of the emerged adults. However our results, obtained in microcosm in a controlled environment with artificially synchronized thrips cohorts, need to be confirmed under greenhouse or field conditions with naturally established and growing thrips populations over longer time intervals. If the additive and synergistic effects of Neem and entomopathogens can be confirmed in the more realistic culture situations, this combination may offer a powerful and reliable tool for thrips control. In addition to combined treatments, sequential treatments and split application of the Entomopathogens may also be an interesting option. That is, if thrips are detected early, neem may provide initial population reduction followed by split application of the Entomopathogens which can offer a long term control of the pupal stage of WFT. More investigation is needed to establish the effect of neem on virulence of entomopathogens since different compatibility studies show some conflicting results. Also studying on the molecular background of innate defence response in WFT after the combined application of neem and the entomopathogens would be interesting.

\section{ACKNOWLEDGEMENT}


The authors would like to thank Mr. Malcolm White and Dr. Theresa Schrem whose comments helped improve the linguistic quality of the manuscript. Many thanks to Dr. Gisela Grunewaldt-Stöcker for helping with the identification of the EPF and to the technical support team of the section Phytomedicine at IGPS, Leibniz Universität Hannover. The study was supported by a grant from German Academic Exchange Service (Deutscher Akademischer Austauschdienst-DAAD).

\section{REFERENCES}

Akbar W, Lord JC, Nechols JR, Loughin TM, 2005: Efficacy of Beauveria bassiana for red flour beetle when applied with plant essential oils or in mineral oil and organosilicone carriers. J Econ Entomol, 98, 683-688.

Ansari MA, Brownbridge M, Shah FA, Butt TM, 2008a: Efficacy of entomopathogenic fungi against soil-dwelling life stages of western flower thrips, Frankliniella occidentalis, in plant-growing media. Entomol Exp Appl 127(2), 80-87.

Ansari MA, Shah FA, Butt TM, 2008b: Combined use of entomopathogenic nematodes and Metarhizium anisopliae as a new approach for black vine weevil, Otiorhynchus sulcatus, control. Entomol Exp Appl 129, 340-347.

Ansari MA, Shah FA, Whittaker M, Prasaad M, Butt TM, 2007: Control of western flower thrips (Frankliniella occidentalis) pupae with Metarhizium anisopliae in peat and peat alternative growing media. Biol Control, 40, 293-297.

Arthurs S, Heinz KM, 2006: Evaluation of the nematodes Steinernema feltiae and Thripinema nicklewoodi as biological control agents of western flower thrips Frankliniella occidentalis infesting chrysanthemum. Biocontrol Sci Techn, 16, 141-155.

Belay D, Ebssa L, Borgemeister C, 2005: Time and frequency of applications of entomopathogenic nematodes and their persistence for control of western flower thrips Frankliniella occidentalis. Nematology, 7(4), 611-622.

Berndt O, 2003: Entomopathogenic nematodes and Soil Dwelling Predatory Mites: Suitable Antagonists for Enhanced Biological Control of Frankliniella occidentalis (Pergande)(Thysanoptera: Thripidae). Doctoral Thesis, Universität Hannover, pp140.

Berndt O, Meyhöfer R, Poehling H-M, 2004: The edaphic phase in the ontogenesis of Frankliniella occidentalis and comparison of Hypoaspis miles and Hypoaspis aculeifer as predators of soil-dwelling thrips stages. Biol Control, 30(1), 17-24. 
Bielza P, Quinto V, Fernández E, Grávalos C, Contreras J, 2007: Genetics of spinosad resistance in Frankliniella occidentalis (Thysanoptera: Thripidae) J Econ Entomol, 100, 916-920.

Bielza P, Quinto V, Grávalos C, Abellán J, Fernández E, 2008: Lack of fitness costs of insecticide resistance in the western flower thrips (Thysanoptera: Thripidae). J Econ Entomol, 101, 499-503.

Boonham N, Smith P, Walsh K, Tame J, Morris J, Spence N, 2002: The detection of Tomato spotted wilt virus (TSWV) in individual thrips using real time fluorescent RT-PCR (TaqMan). J Virol Methods, 101, 37-48.

Brownbridge M, Buitenhuis R, Murphy G, Waite M, Scott-Dupree C, 2013: Banker plants, trap crops and other bioprotection developments in Canadian greenhouse floriculture, In: Mason, P.G., Gillespie, D.R., Vincent, C. (Eds.). In Proc. 4th International Symposium on Biological Control of Arthropods, Pucón, Chile, (pp. 133-136).

Buitenhuis R, Shipp JL, 2005: Efficacy of entomopathogenic nematode Steinernema feltiae (Rhabditida: Steinernematidae) as influenced by Frankliniella occidentalis (Thysanoptera: Thripidae) developmental stage and host plant stage. J Econ Entomol, 98, 1480-1485.

Cloyd RA, 2009: Western Flower Thrips (Frankliniella occidentalis) Management on Ornamental Crops Grown in Greenhouses: Have We Reached an Impasse ? Pest Technology, 3(1), 1-9.

DeCourcy Williams ME, 2001: Biological control of thrips on ornamental crops: Interactions between the predatory mite Neoseiulus cucumeris (Acari:Phytoseiidae) and western flower thrips, Frankliniella occidentalis (Thysanoptera:Thripidae) on cyclamen. Biocontrol Sci.Tech., 11, 41-55.

Desneux N, Denoyelle R, Kaiser L, 2006a: A multi-step bioassay to assess the effect of the deltamethrin on the parasitic wasp Aphidius ervi. Chemosphere 65, 1697-1706.

Desneux N, Fauvergue X, Dechaume-Moncharmont FX, Kerhoas L, Ballanger Y, Kaiser L, 2005: Diaretiella rapae limits Myzus persicae populations after applications of deltamethrin in oilseed rape. J. Econ. Entomol, 98, 9-17. 
Ebssa L, Borgemeister C, Berndt O, Poehling H-M, 2001: Efficacy of entomopathogenic nematodes against soil-dwelling life stages of western flower thrips, Frankliniella occidentalis (Thysanoptera: Thripidae). J Invertebr Pathol, 78(3), 119-127.

Ebssa L, Borgemeister C, Poehling H-M, 2004: Effectiveness of different species/strains of entomopathogenic nematodes for control of western flower thrips (Frankliniella occidentalis) at various concentrations, host densities, and temperatures. Biol Control, 29(1), 145-154.

Gao Y, Lei Z, Reitz SR, 2012: Western flower thrips resistance to insecticides: Detection, mechanisms and management strategies. Pest Manag Sci, 68, 1111-1121.

Georgis R, 1997: Commercial prospects of microbial pesticides in agriculture. In "Microbial Insecticides: Novelty or Necessity” (H.F. Evans, chair). Proc. Br. Crop Prot. Council Symp. 68, 243-252.

Goettel M, Inglis G, 1997: Fungi: hyphomycetes. In LA, Lacey. Manual of techniques in insect pathology (p. 37). Academic Press, San Diego: 213-249

Herrin B, Warnock D, 2002: Resistance of impatiens germplasm to western flower thrips feeding damage. Hort. Science, 37, 802-804.

Herron GA, James TM, 2005: Monitoring insecticide resistance in Australian Frankliniella occidentalis Pergande (Thysanoptera: Thripidae) detects fipronil and spinosad resistance. Aust J. Entomol, 44, 299-303.

Holmes ND, Bennison JA, Maulden KA, Kirk WDJ, 2012: The pupation behaviour of the western flower thrips, Frankliniella occidentalis (Pergande). Acta Phytopathol Hun, 47, 87-96.

Hossain MB,. Poehling H-M, Thöming G, Borgemeister, C, 2007: Effects of soil application of neem (NeemAzal®-U) on different life stages of Liriomyza sativae (Diptera: Agromyzidae) on tomato in the humid tropics. J Plant Dis Protect, 115 (2), 80-87.

Islam M, Castle S, Ren S, 2010: Compatibility of the insect pathogenic fungus Beauveria bassiana with neem against sweetpotato whitefly, Bemisia tabaci, on eggplant. Entomol Exp Appl, 134, 28-34.

Islam MT, Omar D, Latif MA, Morshed M, 2011: The integrated use of entomopathogenic fungus, Beauveria bassiana with botanical insecticide, neem against Bemisia tabaci on eggplant. African J Microbiol Res, 5,3409- 3413. 
Jacobson RJ, Chandler D, Fenlon J, Russel KM, 2001: Compatibility of Beauveria bassiana (Balsamo) Vuillemin with Amblyseius cucumeris Oudemans (Acarina: Phytoseiidae) to control Frankliniella occidentalis Pergande (Thysanoptera: Thripidae) on cucumber plants. Biocontrol Sci Tech, 11, 391- 400.

James RR, 2003: Combining azadirachtin and Paecilomyces fumosoroseus (Deuteromycotina: Hyphomycetes) to control Bemisia argentifolii (Homoptera: Aleyrodidae). J Econ Entomol, 96, 25-30.

Kaya HK, Stock SP, 1997: Techniques in insect nematology. In: Lacey, L. (Ed.) Manual of techniques in insect pathology. Academic Press Ltd. San Diego, California, (pp. 281324.).

Krishnayya PV, Grewal PS, 2002: Effect of neem and Selected fungicides on viability and virulence of the Entomopathogenic nematode Steinernema feltiae. Biocontrol Sci Techn, 12, 259-266.

Kumar P, Singh HP, Poehling H-M, 2010: Effects of neem on adults of Eretmocerus warrae (Hym. Aphelinidae), a parasitoid of Bemisia tabaci (Hom. Aleyrodidae) in tropical horticulture systems. J Plant Dis Protect, 117 (6), 273-277.

Lacey L, Frutos R, Kaya H, Vail P, 2001: Insect Pathogens as Biological Control Agents: Do They Have a Future? Biol Control, 21(3), 230-248.

Lee K-Y, Lynn OM, Song W-G, Shim J-K,Kim J-E, 2010: Effects of Azadirachtin and Neem-based Formulations for the Control of Sweet potato Whitefly and Root-knot Nematode. J. Korean Soc. Appl Biol Chem, 53(5), 598-604.

MahMoud M, 2007: Combining the botanical insecticides NSK extract, NeemAzal T 5\%, Neemix 4.5\% and the entomopathogenic nematode Steinernema feltiae Cross N 33 to. Plant Protection Science-Prague 43(1), 19-25.

McCullagh P, Nelder JA, 1989: Generalized Linear Models, Second Edition, Chapman \& Hall/CRC, Boca Raton, FL.

Meyer J, Ebssa L, Poehling H-M, 2012: Survival, host infestation and reproduction of entomopathogenic and plant-parasitic nematodes: Heterorhabditis bacteriophora and Meloidogyne incognita. J Plant Dis Protect, 119(4), 142-151. 
Mitchell PL, Gupta R, Singh AK, Kumar P, 2004: Behavioural and developmental effects of neem extarcts on Clavigralla scutellaris (Hemiptera: Heteroptera: Coreidae) and its egg parasitoid, Gryon fulviventre (Hymenoptera: Scelionidae). J Econ Entomol, 97, 916-923

Mohan MC, Reddy NP, Devi UK, Ramesh K, Sharma HC, 2007: Growth and insect assays of Beauveria bassiana with neem to test their compatibility and synergism. Biocontrol Sci Techn, 17(10), 1059-1069.

Morse JG, Hoddle MS, 2006: Invasion Biology of Thrips. Annu Rev Entomol, 51, 67-89.

Niassy S, Maniania NK, Subramanian S, Gitonga LM, Maranga R, Obonyo AB, Ekesi S, 2012a: Compatibility of Metarhizium anisopliae isolate ICIPE 69 with agrochemicals used in French bean production. Int. J. Pest. Manage, 58 (2) 131-137.

Niassy S, Maniania NK, Subramanian S, Gitonga LM, Mburu DM, Masiga D, Ekesi S, 2012b: Selection of promising fungal biological control agent of the western flower thrips Frankliniella occidentalis (Pergande). Letters in Applied Microbiology, 54 (6) 487-493.

Pappu HR, Jones RAC, Jain RK, 2009: Global status of tospovirus epidemics in diverse cropping systems: Successes achieved and challenges ahead, Virus Res, 141(2), 219236.

Premachandra WTSD, Borgemeister C, Berndt O, Ehlers R-U, Poehling H-M, 2003a: Combined releases of entomopathogenic nematodes and the predatory mite Hypoaspis aculeifer to control soil-dwelling stages of western flower thrips Frankliniella occidentalis. Biol Control, 48, 529-541.

Premachandra D, Borgemeister C, Berndt O, Ehlers UE, Poehling H-M, 2003b: Laboratory bioassays of virulence of entomopathogenic nematodes against soil-inhabiting stages of Frankliniella occidentalis Pergande (Thysanoptera: Thripidae). Nematology, 5(4), 539547.

R Core Team, 2014: R: A Language and Environment for Statistical Computing, R Foundation for Statistical Computing, Vienna, Austria. http://www.R-project.org/.

Reitz S, 2009: Biology and ecology of the western flower thrips (Thysanoptera: Thripidae): The making of a pest. Florida Entomologist, 92, 7-13.

Riley DG, Pappu HR, 2004: Tactics for management of thrips (Thysanoptera: Thripidae) and tomato spotted wilt virus in tomato. J Econ Entomol, 97, 1648-1658. 
Schmutterer H, 1997: Side-effects of neem (Azadirachta indica) products on insects pathogens and natural enemies of spider mites and insects. J Appl Entomol, 21, 121-128.

Shan C, Ma S, Wang M, Gao G, 2012: Evaluation of insecticides against the western flower thrips, Frankliniella occidentalis (Thysanoptera: Thripidae), in the laboratory. Florida Entomologist, 95, 454-460.

Shapiro-Ilan DI, Han R, Dolinksi C, 2012: Entomopathogenic nematode production and application technology. J Nematol, 44(2), 206-17.

Shipp JL, Wang K, Binns M, 2000: Economic injury level western flower thrips (Thysanoptera: Thripidae) on greenhouse cucumber. J Econ Entomol, 93, 1732-1740.

Skinner M, Gouli S, Frank CE, Parker BL, Kim JS 2012: Management of Frankliniella occidentalis (Thysanoptera: Thripidae) with granular formulations of entomopathogenic fungi. Biol Control 63, 246-252.

Steiner MY, Spohr LJ, Goodwin S, 2011: Relative humidity controls pupation success and dropping behaviour of western flower thrips, Frankliniella occidentalis (Pergande) (Thysanoptera: Thripidae). Aust J Entomol, 50, 179-186.

Thoeming G, Draeger G, Poehling H-M, 2006: Soil application of azadirachtin and 3-tigloylazadirachtol to control western flower thrips, Frankliniella occidentalis (Thysanoptera: Thripidae): translocation and persistence in bean plants. Pest Manag Sci, 62, 759-767.

Thoeming G, Borgemeister C, Sétamou M, Poehling H-M, 2003: Systemic Effects of Neem on Western Flower Thrips, Frankliniella occidentalis (Thysanoptera: Thripidae ). J Econ Entomol, 96(3), 817-825.

Thoeming G, Poehling H-M, 2006: Soil application of different neem products to control Ceratothripoides claratris (Thysanoptera: Thripidae) on tomatoes grown under protected cultivation in the humid tropics Thailand. Int J Pest Manage, 52(3), 239-248.

Thoeming G, 2005: Soil application of neem products in IPM: Controlling thrips (Thysanoptera: Thripidea) in vegetables crops. University Of Hannover, Germany. Doctoral Thesis, 119 pp.

Webster CG, Reitz SR, Perry KL, Adkins S, 2011: A natural M RNA reassortant arising from two species of plant-and insect-infecting bunyaviruses and comparison of its sequence and biological properties to parental species. Virology, 413, 216-225. 
Williams C, Dillon A, Harvey C, 2013: Control of a major pest of forestry, Hylobius abietis, with entomopathogenic nematodes and fungi using eradicant and prophylactic strategies.

Forest Ecol Manag, 305, 212-222.

\section{Legend of Figures}

\section{Fig. 1 Left -}

Emergence of western flower thrips (mean +/- SD) in single treatments of Neem Azal-T solution (1\% Azadirachtin A) and Neem pellets (7 \% Azadirachtin); Beauveria bassiana, Metarhizium anisopliae (IPP 2539 \& ICIPE-69) and Steinernema carpocapsae.

Same letters indicate significant differences at a multiple type I error level of 5\% (Tukey’s test).

Right- Trend lines showing daily emergence of western flower thrips within the seven-day period

Black arrow- Peak of adult emergence course beyond which emergence steadily reduced.

\section{Fig. 2 Left -}

Emergence of western flower thrips (mean +/- SD) in combined treatments amongst Neem AzalT solution (1\% Azadirachtin A); Neem pellets (7 \% Azadirachtin; Metarhizium anisopliae ICIPE-69 (EPF) and Steinernema carpocapsae (EPN).

Treatments with different letters are significantly different at a multiple type I error level of 5\% (Tukey's test).

Right- Trend lines showing daily emergence of western flower thrips within the seven day period

Black arrow- Peak of adult emergence course beyond which emergence steadily reduced.

\section{Fig. 3}

Emergence and survivors of western flower thrips (mean +/- SD) in EPF based single and combined treatments (Beauveria bassiana, Metarhizium anisopliae (IPP 2539 \& ICIPE-69). Number of adults is given as total number of emergences (light gray) and number of survivors taking late mortality by mycosis into account (dark gray).

Different letters indicate significant differences at a multiple type I error level of 5\% (Tukey's test).

\section{Fig. 4}


Emergence of western flower thrips (mean +/- SD) in combinations of three level doses of Neem Azal-T solution (1\% Azadirachtin A); Metarhizium anisopliae (ICIPE-69) and Steinernema carpocapsae

Different letters indicate significant differences at a multiple type I error level of 5\% (Tukey's test). 

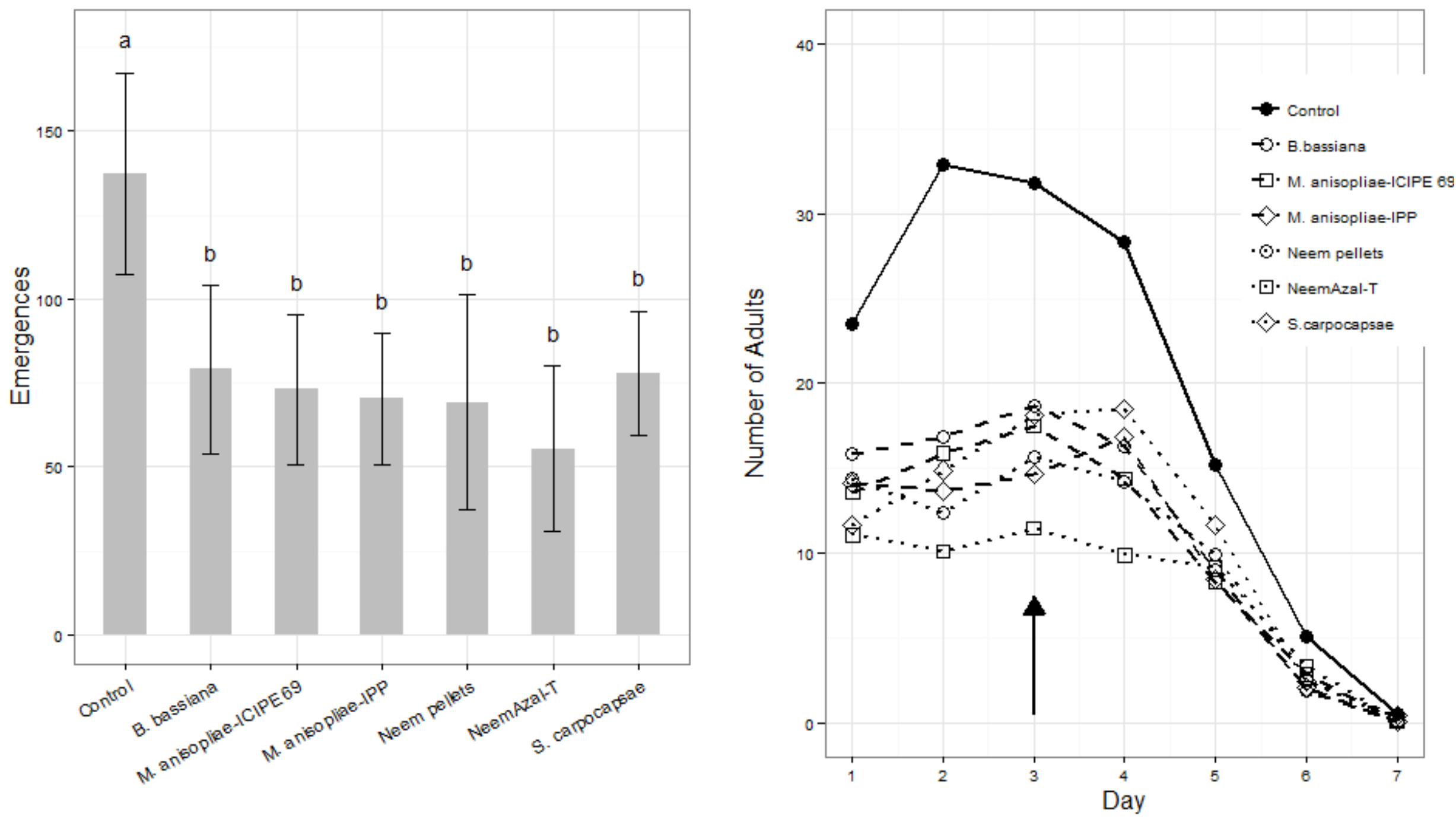

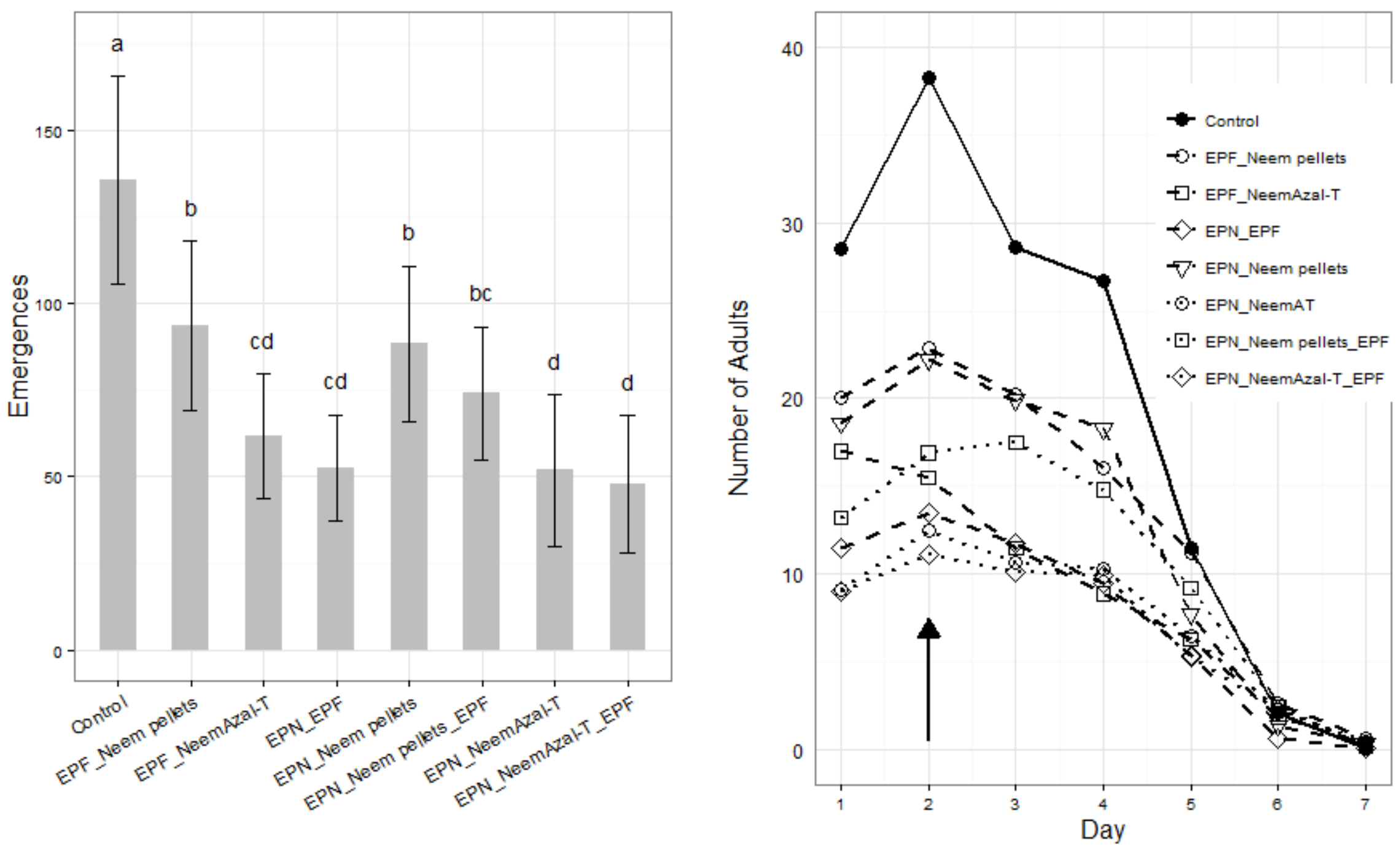


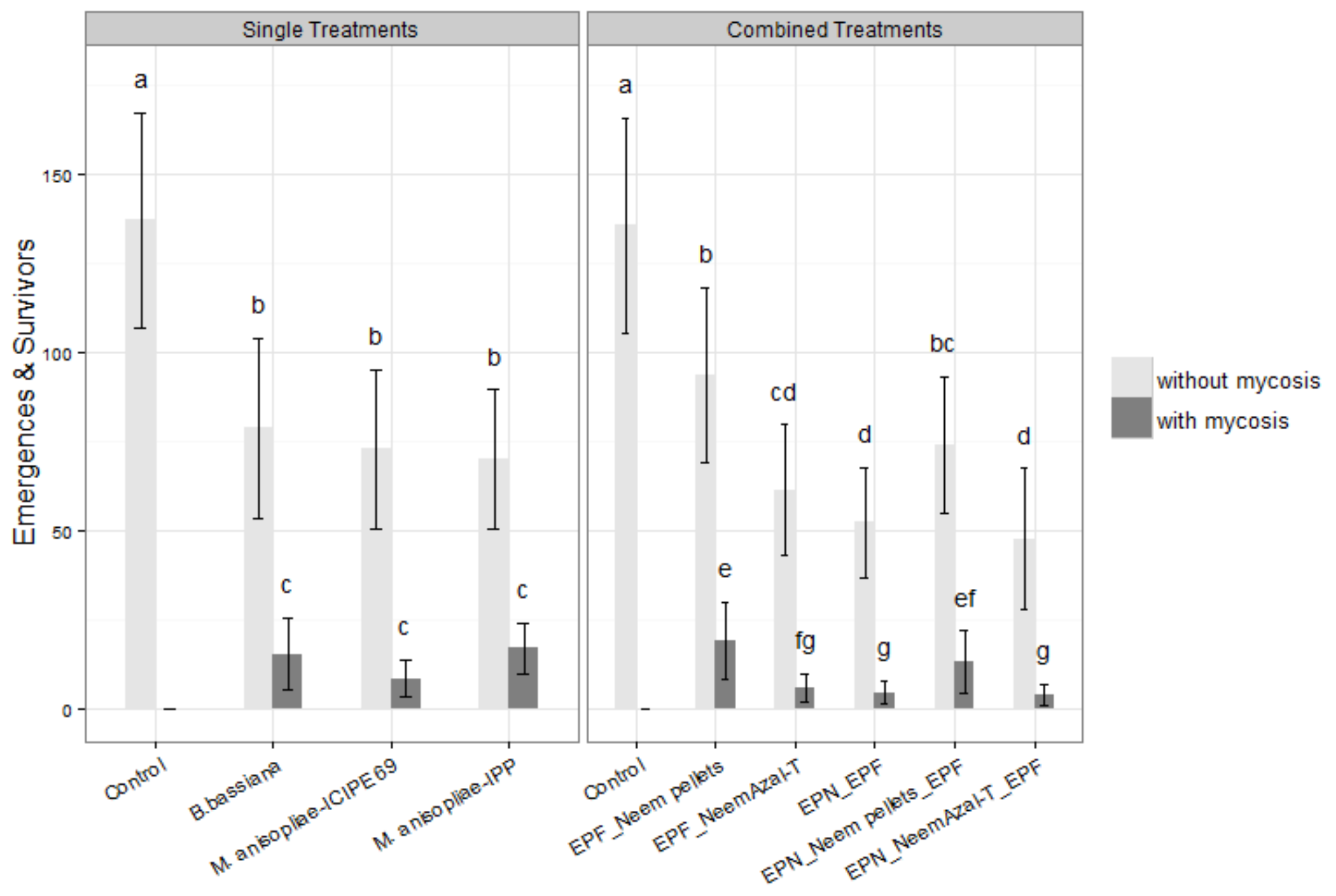




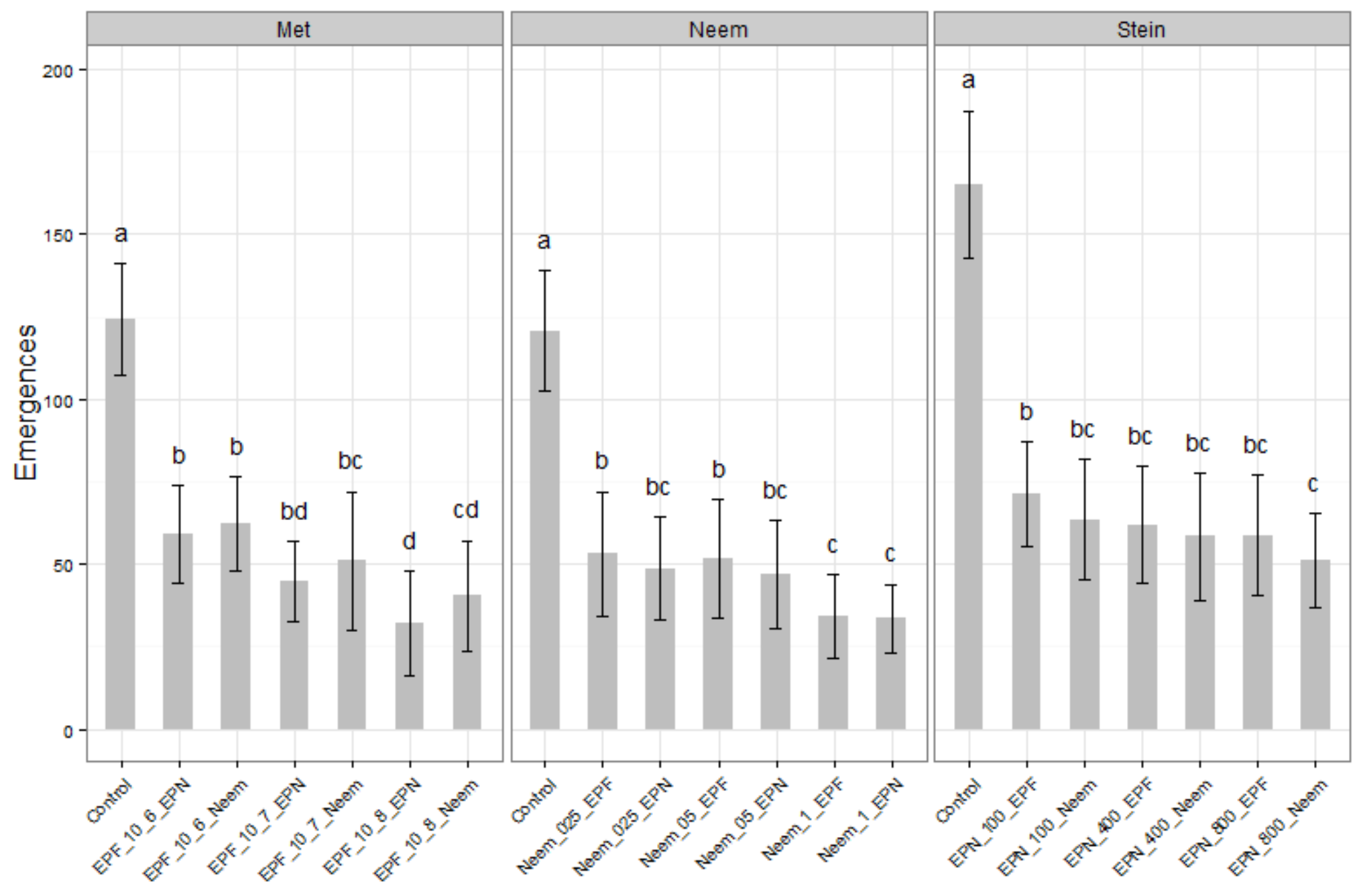


Table S1: Applied doses of M. anisopliae-ICIPE, Neem Azal-T, Neem pellets, and S. carpocapsae for the single treatments and combinations, given as amount of active compounds/pot (600g of soil) and percentages (relative to the single treatment doses).

\begin{tabular}{|c|c|c|c|c|c|}
\hline \multirow[t]{7}{*}{$\mathbf{A}$} & Treatment(s) & $\begin{array}{l}\text { M. anisopliae- } \\
\text { ICIPE (EPF) }\end{array}$ & NeemAzal-T & Neem pellets & $\begin{array}{l}\text { S. carpocapsae } \\
\text { (EPN) }\end{array}$ \\
\hline & Single treatments & & & & \\
\hline & M. anisopliae-ICIPE (EPF) & $10^{7}$ conidia & - & - & - \\
\hline & NeemAzal-T & - & $\begin{array}{c}6 \mathrm{mg} \\
\text { Azadirachtin A }\end{array}$ & - & - \\
\hline & Neem pellets & - & - & $\begin{array}{c}6 \mathrm{mg} \\
\text { Azadirachtin A }\end{array}$ & - \\
\hline & S. carpocapsae (EPN) & - & - & - & $\begin{array}{c}80435 \mathrm{IJ} \\
\left(400 \mathrm{IJ} / \mathrm{cm}^{2}\right)\end{array}$ \\
\hline & Double combinations & $\%$ of single treat & used-See A & & \\
\hline \multirow[t]{3}{*}{ B } & EPF + NeemAzal-T & $(40 \%)$ & $(60 \%)$ & - & - \\
\hline & EPF + Neem pellets & $(50 \%)$ & - & $(50 \%)$ & - \\
\hline & $\mathrm{EPF}+\mathrm{EPN}$ & $(67 \%)$ & - & - & $(33 \%)$ \\
\hline & NeemAzal-T + EPN & - & $(75 \%)$ & - & $(25 \%)$ \\
\hline & Neem pellets + EPN & - & - & $(67 \%)$ & $(33 \%)$ \\
\hline & Triple combination & $\%$ of single treat & used-See A & & \\
\hline \multirow[t]{2}{*}{$\mathrm{C}$} & EPF + NeemAzal-T + EPN & $(33 \%)$ & $(50 \%)$ & - & $(17 \%)$ \\
\hline & EPF + Neem pellets + EPN & $(40 \%)$ & - & $(40 \%)$ & $(20 \%)$ \\
\hline
\end{tabular}

Table 1: Evaluation of treatment combinations: estimated percent deviations from additivity with $95 \%$ confidence intervals, p-values, and conclusions about interactions based on quasiPoisson GLM analysis.

\begin{tabular}{lcccc}
\hline $\begin{array}{l}\text { Treatment } \\
\text { combination }\end{array}$ & $\begin{array}{c}\text { Estimated \% deviation } \\
\text { from additivity }\end{array}$ & $\begin{array}{c}\text { 95\% confidence } \\
\text { interval }\end{array}$ & $\begin{array}{c}\text { p- } \\
\text { value }\end{array}$ & Interaction \\
\hline EPF + NeemAzal-T & $-0.9 \%$ & $-24.4 \% ;+30.0 \%$ & 0.949 & additive \\
EPF + Neem pellets & $-25.0 \%$ & $-41.3 \% ;-4.2 \%$ & 0.022 & antagonistic \\
EPF + EPN & $+40.4 \%$ & $+6.4 \% ;+85.2 \%$ & 0.016 & synergistic \\
NeemAzal-T + EPN & $+14.8 \%$ & $-14.0 \% ;+53.2 \%$ & 0.351 & additive \\
Neem pellets + EPN & $-19.6 \%$ & $-37.4 \% ;+3.3 \%$ & 0.088 & additive \\
EPF + NeemAzal-T + & $+32.7 \%$ & $+0.4 \% ;+75.5 \%$ & 0.047 & synergistic \\
EPN & & & & \\
EPF + Neem pellets + & $-3.5 \%$ & $-24.8 \% ;+23.7 \%$ & 0.776 & additive \\
EPN & & & & \\
\hline
\end{tabular}

